\title{
Recursive Computation of the Repeated Integrals of the Error Function
}

\section{By Walter Gautschi}

1. This paper is concerned with a special technique, originated by J. C. P. Miller [1, p. xvii], of computing a solution $f_{n}$ of a second-order difference equation

$$
y_{n+1}+a_{n} y_{n}+b_{n} y_{n-1}=0 \quad(n=1,2,3, \cdots)
$$

for $n=0(1) N, N$ large, in cases where (1.1) has a second solution, $g_{n}$, which ultimately grows much faster than $f_{n}$. Straightforward use of (1.1) is then not adequate, since rounding errors will "activate" the second solution $g_{n}$, which in turn will eventually overshadow the desired solution $f_{n}$. Miller's device consists of applying (1.1) in backward direction,

$$
y_{n-1}=-b_{n}^{-1}\left(a_{n} y_{n}+y_{n+1}\right) \quad(n=\nu-1, \nu-2, \cdots, 1 ; \nu>N),
$$

starting with the initial values

$$
y_{\nu-1}=\alpha, \quad y_{\nu}=0,
$$

where $\alpha$ is any real number $\neq 0$. If $\nu$ is taken sufficiently large the values so obtained turn out to be approximately proportional to $f_{n}$ in the range $0 \leqq n \leqq N$. The factor of proportionality may then be determined, e.g., by comparing $y_{0}$ with $f_{0}$.

This technique was originally devised [1] for the computation of Bessel functions $I_{n}(x)$, and has since then been applied to various other Bessel functions [2], [5], [9], to Legendre functions [8], and to the repeated integrals of the error function* [6],

$$
\begin{gathered}
i^{n} \operatorname{erfc} x=\frac{2}{\sqrt{\pi}} \int_{x}^{\infty} \frac{(t-x)^{n}}{n !} e^{-t^{2}} d t \quad(n=0,1,2, \cdots), \\
i^{-1} \operatorname{erfc} x=\frac{2}{\sqrt{\pi}} e^{-x^{2}}:
\end{gathered}
$$

An analogous technique for first-order difference equations is described in $[4$, p. 25].

We shall present in Section 2 a detailed description of Miller's procedure, paying special attention to the error term. In Section 3 we study the procedure as applied to the computation of the functions (1.4) and show that the process converges for any positive $x$, as $\nu \rightarrow \infty$. In Sections 4-5 estimates will be developed of how large $\nu$ must be taken to ensure any prescribed accuracy.

Received August 22, 1960.

* In this notation $i^{n}(n \geqq 0)$ should be interpreted as the $n$th power of the integral operator $i=\int_{x}^{\infty}$, so that

$$
i^{0} \operatorname{erfc} x=\operatorname{erfc} x, \quad i^{n} \operatorname{erfc} x=\int_{x}^{\infty} i^{n-1} \operatorname{erfc} t d t \quad(n=1,2, \cdots) .
$$

This notation for the repeated integrals of the error function, even though not entirely satisfactory, has become standard. 
2. Consider the homogeneous second-order difference equation

$$
y_{n+1}+a_{n} y_{n}+b_{n} y_{n-1}=0 \quad(n=1,2,3, \cdots)
$$

and assume that

$$
b_{n} \neq 0 \text { for all } n \geqq 1 \text {. }
$$

Let $f_{n}$ be the (nontrivial) solution of (2.1) to be computed for $n=0(1) N$. We assume

$$
f_{0} \neq 0 \text {. }
$$

Let there be another solution $g_{n}$ of $(2.1)$, for which

$$
g_{n} \neq 0 \text { for all } n \geqq 0 \text {, }
$$

and

$$
\lim _{n \rightarrow \infty}\left|\frac{f_{n}}{g_{n}}\right|=0 .
$$

It follows readily that $f_{n}, g_{n}$ are linearly independent.

Now let $y_{n}^{(\nu)}(n=0,1, \cdots, \nu-2 ; \nu>N)$ be the result of applying (2.1) in backward direction, starting with

$$
y_{v-1}^{(\nu)}=\alpha, \quad y_{\nu}^{(v)}=0 \quad(\alpha \neq 0) .
$$

These values, by (2.2), are well defined, and, as will presently be shown, $y_{0}^{(v)} \neq 0$ for $\nu$ sufficiently large. Let us then define

$$
f_{n}^{(v)}=\frac{f_{0}}{y_{0}{ }^{(v)}} y_{n}^{(v)}
$$

We show that for any fixed $n$,

$$
\lim _{v \rightarrow \infty} \dot{f}_{n}^{(v)}=f_{n}
$$

Moreover,

$$
f_{n}^{(v)}=f_{n} \frac{1-\frac{f_{\nu}}{g_{\nu}} \frac{g_{n}}{f_{n}}}{1-\frac{f_{\nu}}{g_{\nu}} \frac{g_{0}}{f_{0}}} .
$$

It is sufficient to prove (2.9), since (2.8) then follows from (2.5). Let $y_{n}^{(v)}$ be extended to all $n>\nu$ by means of (2.1). Then for every fixed $\nu$ the sequence $\left\{y_{n}{ }^{(v)}\right\}(n=0,1,2, \cdots)$ is a solution of (2.1), and therefore representable in the form

$$
y_{n}^{(v)}=A^{(v)} f_{n}+B^{(v)} g_{n} \quad(n \geqq 0) .
$$

By (2.6),

$$
\begin{aligned}
A^{(v)} f_{v-1}+B^{(v)} g_{v-1} & =\alpha, \\
A^{(v)} f_{\nu}+B^{(v)} g_{\nu} & =0 .
\end{aligned}
$$


Certainly, $A^{(v)} \neq 0$, since otherwise, by $(2.4), A^{(v)}=B^{(v)}=0$, which contradicts the first equation in (2.10). From the second equation, $B^{(v)} / A^{(v)}=-f_{v} / g_{\nu}$. Therefore

$$
y_{n}^{(\nu)}=A^{(v)}\left(f_{n}+\frac{B^{(\nu)}}{A^{(\nu)}} g_{n}\right)=A^{(v)}\left(f_{n}-\frac{f_{\nu}}{g_{\nu}} g_{n}\right) \text {. }
$$

If $\nu$ is sufficiently large it follows because of (2.3) and (2.5) that $y_{0}{ }^{(v)} \neq 0$. By (2.7),

$$
f_{n}^{(\nu)}=\frac{f_{0} A^{(v)}\left(f_{n}-\frac{f_{\nu}}{g_{\nu}} g_{n}\right)}{A^{(v)}\left(f_{0}-\frac{f_{\nu}}{g_{\nu}} g_{0}\right)}=f_{n} \frac{1-\frac{f_{\nu}}{g_{\nu}} \frac{g_{n}}{f_{n}}}{1-\frac{f_{\nu}}{g_{\nu}} \frac{g_{0}}{f_{0}}}
$$

which proves (2.9).

It is convenient to define

$$
\rho_{n}=\frac{f_{n} g_{0}}{g_{n} f_{0}} \quad(n=0,1,2, \cdots)
$$

so that $\rho_{n} \rightarrow 0$ as $n \rightarrow \infty$, and

$$
f_{n}{ }^{(\nu)}=f_{n} \frac{1-\left(\rho_{\nu} / \rho_{n}\right)}{1-\rho_{\nu}} .
$$

The relative error of $f_{n}^{(\nu)}$ is given by

$$
\frac{f_{n}^{(\nu)}-f_{n}}{f_{n}}=\frac{\rho_{\nu}}{1-\rho_{\nu}}\left(1-\frac{1}{\rho_{n}}\right) \text {. }
$$

The approximations $f_{n}{ }^{(\nu)}$ obviously do not depend on $\alpha$, so that $\alpha$ can be chosen at will. If a high-speed computer is employed it is advisable to choose a small value for $\alpha$ to guard against "overflow" in the values of $y_{n}{ }^{(v)}$.

3. Now let

$$
f_{n}=i^{n-1} \operatorname{erfc} x, \times 70 \quad(n=0,1,2, \cdots) .
$$

Then $f_{n}$ is a solution of

$$
y_{n+1}+\frac{x}{n} y_{n}-\frac{1}{2 n} y_{n-1}=0 \quad(n=1,2,3, \cdots)
$$

as is readily verified by writing

$$
i^{n} \operatorname{erfc} x=\frac{2}{\sqrt{\pi}}\left(\frac{1}{n} \int_{x}^{\infty} \frac{(t-x)^{n-1}}{(n-1) !} t e^{-t^{2}} d t-\frac{x}{n} \int_{x}^{\infty} \frac{(t-x)^{n-1}}{(n-1) !} e^{-t^{2}} d t\right)
$$

and evaluating the first integral by parts. A second solution of (3.2) is given by

$$
g_{n}=(-1)^{n} i^{n-1} \operatorname{erfc}(-x)
$$$$
(n=0,1,2, \cdots) \text {. }
$$

It is clear that the assumptions (2.2)-(2.4) are satisfied in this case. We shall now verify (2.5), i.e.

$$
\lim _{n \rightarrow \infty} \frac{i^{n} \operatorname{erfc} x}{i^{n} \operatorname{erfc}(-x)}=0 \quad(x>0) .
$$


This then will prove the convergence of the procedure in Section 2, as applied to (3.2).

We recall that the repeated integrals of the error function are related to the parabolic cylinder functions $D_{\nu}(x)$ by $[7$, p. 76]

$$
i^{n} \operatorname{erfc} x=\frac{e^{-\frac{1}{2} x^{2}}}{2^{(n-1) / 2} \sqrt{\pi}} D_{-n-1}(x \sqrt{2})
$$

It is furthermore known [3, p. 123] that

$$
D_{-n-1}(z)=\frac{\sqrt{\pi} e^{-\sqrt{n} z}}{2^{(n+1) / 2} \Gamma\left(\frac{n}{2}+1\right)}\left[1+O\left(\frac{1}{\sqrt{n}}\right)\right] \quad(n \rightarrow \infty, z \text { bounded }) .
$$

Therefore we obtain immediately for any fixed $x$, real or complex,

$$
i^{n} \operatorname{erfc} x=\frac{e^{-\frac{1}{2} x^{2}}}{2^{n} \Gamma\left(\frac{n}{2}+1\right)} e^{-\sqrt{2 n} x}\left[1+O\left(\frac{1}{\sqrt{n}}\right)\right] \quad(n \rightarrow \infty)
$$

Hence,

$$
\frac{i^{n} \operatorname{erfc} x}{i^{n} \operatorname{erfc}(-x)} \sim e^{-2 \sqrt{2 n} x} \quad(n \rightarrow \infty)
$$

which proves (3.4).

4. With $f_{n}, g_{n}$ defined by (3.1) and (3.3) we have for the quantities $\rho_{n}$ in (2.11)

$$
\rho_{n}=(-1)^{n} \frac{i^{n-1} \operatorname{erfc} x}{i^{n-1} \operatorname{erfc}(-x)} \quad(n=0,1,2, \cdots) .
$$

It is shown in this section that for any fixed $x>0$ the sequence $\left\{\left|\rho_{n}\right|\right\}$ is monotonically decreasing, i.e.,

$$
\left|\frac{\rho_{n+1}}{\rho_{n}}\right|=\frac{i^{n} \operatorname{erfc} x}{i^{n-1} \operatorname{erfc} x} \cdot \frac{i^{n-1} \operatorname{erfc}(-x)}{i^{n} \operatorname{erfc}(-x)}<1 \quad(n \geqq 0) .
$$

Inequality (4.2) is obvious if $n=0$ and, by (1.4), equivalent to

$$
\begin{aligned}
\int_{x}^{\infty}(t-x)^{n-1} e^{-t^{2}} d t \int_{-x}^{\infty}(s+x)^{n} e^{-s^{2}} d s & \\
& -\int_{x}^{\infty}(t-x)^{n} e^{-t^{2}} d t \int_{-x}^{\infty}(s+x)^{n-1} e^{-s^{2}} d s>0
\end{aligned}
$$

if $n>0$. By introducing new variables of integration, $t=u+x, s=v-x$, and writing the left-hand side as a double integral, one obtains

$$
\iint_{Q} u^{n-1} v^{n-1}(v-u) e^{-(u+x)^{2}-(v-x)^{2}} d u d v>0,
$$

where $Q$ denotes the first quadrant $u \geqq 0, v \geqq 0$. Let $Q_{1}, Q_{2}$ denote the regions

$$
Q_{1}: \quad 0<u<v, \quad Q_{2}: \quad 0<v<u \text {. }
$$


Interchanging variables of integration gives

$\iint_{\mathbf{Q}_{2}} u^{n-1} v^{n-1}(v-u) e^{-(u+x)^{2}-(v-x)^{2}} d u d v=-\iint_{\mathbf{Q}_{1}} u^{n-1} v^{n-1}(v-u) e^{-(v+x)^{2}-(u-x)^{2}} d u d v$.

Therefore (4.3) is equivalent to

$$
\iint_{Q_{1}} u^{n-1} v^{n-1}(v-u)\left[e^{-(u+x)^{2}-(v-x)^{2}}-e^{-(v+x)^{2}-(u-x)^{2}}\right] d u d v>0 .
$$

Now, $u^{n-1} v^{n-1}(v-u)>0$ in $Q_{1}$, and the same is true for the expression in brackets, since

$$
-(u+x)^{2}-(v-x)^{2}>-(v+x)^{2}-(u-x)^{2} \text { for } u<v .
$$

This proves (4.3), and thus (4.2).

5. We are now in a position to estimate $\nu$ such that for any given integer $p$,

$$
\left|\left(f_{n}{ }^{(\nu)}-f_{n}\right) / f_{n}\right| \leqq 10^{-p} \text { for } n=0,1, \cdots, N+1 .
$$

Here, $f_{n}{ }^{(\nu)}$ denotes the approximations to $f_{n}=i^{n-1}$ erfc $x$ obtained by the procedure of Section 2.

Since, by (2.12),

$$
\left|\left(f_{n}{ }^{(\nu)}-f_{n}\right) / f_{n}\right| \leqq\left|\rho_{\nu}\right|\left(1+\left|\rho_{n}\right|^{-1}\right)+0\left(\rho_{\nu}{ }^{2}\right),
$$

and since $\left|\rho_{n}\right|^{-1}$ increases with $n$, by (4.2), it is sufficient to choose $\nu$ such that

$$
\left|\rho_{\nu}\right|\left(1+\left|\rho_{N+1}\right|^{-1}\right) \leqq 10^{-p} \text {. }
$$

From (3.5) and (4.1) we have

$$
\left|\rho_{n+1}\right|=e^{-2 \sqrt{2 n} x}\left[1+O\left(\frac{1}{\sqrt{ } \bar{n}}\right)\right] .
$$

Assuming $N$ large enough to neglect the $O$-term in (5.2) for $n \geqq N$, the requirement (5.1) may be simplified to

$$
e^{-2 \sqrt{2 \nu} x} \leqq \frac{10^{-p}}{1+e^{2 \sqrt{2 N} x}}
$$

or even to

$$
e^{-2 \sqrt{2 \nu} x} \leqq \frac{10^{-p}}{2 e^{2 \sqrt{2 N} x}}
$$

having made the right-hand bound smaller. Inequality (5.3) yields

$$
\nu \geqq\left(\frac{2 \sqrt{2 N} x+p \ln 10+\ln 2}{2 \sqrt{2} x}\right)^{2},
$$

which gives us the desired estimate of $\nu$.

Oak Ridge National Laboratory

Oak Ridge, Tennessee 
1. British Association for the Advancement of Science, Mathematical Tables, Volume $X$, Bessel Functions, Part II, Functions of Positive Integer Order, Cambridge University Press, 1952.

2. F. J. Corbat6 \& J. L. URETsky, "Generation of spherical Bessel functions in digital computers," J. Assoc. Comp. Mach., v. 6, 1959, p. 366-375.

3. A. ERdelyi, ET al., Higher Transcendental Functions, Vol. II, McGraw-Hill Book Co., Inc., New York, 1953.

4. W. Gautschi, "Recursive Computation of certain integrals," J. Assoc. Comput. Mach., v. 8, 1961, p. 21-40.

5. M. Goldstein \& R. M. Thaler, "Recurrence techniques for the calculation of Bessel functions," $M T A C$, v. 13, 1959, p. 102-108.

6. J. KAYE, "A table of the first eleven repeated integrals of the error function," J. Math. Phys., v. 34, 1955, p. 119-125. See also RMT 58, MTAC, v. 10, 1956, p. 176.

7. National Physical Laboratory, Tables of Weber Parabolic Cylinder Functions, J. C. P. Miller, Editor, H. M. Stationery Office, London, 1955.

8. A. Roten Berg, "The calculation of toroidal harmonics," Math. Comp., v. 14, 1960, p. 274-276.

9. I. A. Stegun \& M. Abramowitz, "Generation of Bessel functions on high-speed computers," $M T A C$, v. 11, 1957, p. 255-257. 